\title{
Validating the Selection Process of Healthy Control Group Based on Apparently Healthy Volunteers
}

\section{Naji SA1, Mahmood MM1* , Alnuaimi AS² and Yousif RW1 \\ ${ }^{1}$ Department of Biology, Mustansiriyah University, Iraq \\ ${ }^{2}$ Baghdad Medical College, University of Baghdad, Iraq}

*Corresponding author: Majid Mohammed Mahmood, Department of Biology, College of Science, Mustansiriyah University, Baghdad, Iraq, Email: Majidmahmood93@yahoo.com

\section{Research Article \\ Volume 3 Issue 3}

Received Date: September 03, 2019

Published Date: September 16, 2019

DOI: $10.23880 /$ oajpr-16000185

\section{Abstract}

Objectives: The objective of this cross sectional study is to evaluate the probability of finding healthy control subjects according to the results of multiple lab tests in multiple domains including biochemical hematological and immunological measurements.

Material and methods: During the period March- June 2016 a sample of 217 apparently healthy Iraqi adults were investigated whether or not they satisfactorily meet the criteria accomplishing the reality of being healthy. Blood specimens were collected from each participant using standard procedures. The following measurements and tests were carried out for all studied participants: anthropometric measurements, complete blood picture test, enzymatic colorimetric assay for serum lipid profile, glucose, urea, creatinine, alanine transferase, and Enzyme Linked Immunosorbent Assay (ELISA) for serum high sensitive $\mathrm{C}$ reactive protein and interleukin 1 beta.

Results: The prevalence rates (PR) of apparently healthy individuals (AHI) were in descending order of wellness requirements as follow: in those with a completely normal lipid profile it was $25.3 \%$, for biochemistry domain $31.3 \%$, for white blood cell count domain 57.2\%, for red blood cell count (RBC) domain $11.5 \%$ and for platelets domain tests it was 31.3\%. A completely normal hematologic domain tests was found in only 8.8\% of tested individuals, while for immunologic domain 9.2\%. The probability of finding a normal control subject based on multiple testing domains was as low as $13.4 \%$.

Conclusions: Avery considerable proportion of population who appear to be healthy, are not in reality, accordingly not all apparently healthy controls are qualified as eligible control. The really healthy control subject is of low probability $(13.4 \%)$ among Iraqi apparently healthy adults. The WBC domain ranked at the top of restriction normality pyramid, followed by biochemistry, lipid profile, RBC domain and immunological domain respectively.

Keywords: Reference intervals; Apparently healthy individual; Control group 


\section{Open Access Journal of Pharmaceutical Research}

Abbreviations: RIs: Reference Intervals; RV: Reference Values; ELISA: Enzyme Linked Immunosorbent Assay; PR: Prevalence Rates; AHI: Apparently Healthy Individuals; TC: Total cholesterol; HDCL: High Density Lipoprotein Cholesterol; LDCL: Low Density Lipoprotein Cholesterol; LDN: Labor Diagnostika Nord.

\section{Introduction}

Correct interpretation of laboratory tests is a major concern for physicians and medical laboratories. Accurately validated reference intervals (RIs) for each quantitative test is one of the main criteria for medical decision. Reference values (RV) are used to define the dispersion of variables in healthy individuals. RVs first introduced as a philosophy, have gained worldwide acceptance as one of the most influential tools in laboratory medicine to help in the clinical management process [1-3]. RI is defined as the interval between two reference limits (these included).

Reference subjects are generally assumed to be "healthy"; however, health is relative and lacks a precise and quantifiable definition. Therefore, reference individuals are selected using "well-defined criteria" i.e., inclusion and exclusion criteria, which approximate health. Such criteria should be defined specifically, according to the goals of the study, and may differ from one study to another. Strictly derived reference ranges are critical for differentiating healthy from diseased individuals and constitute the foundation of our contemporary methodology to making the diagnosis of clinical disorders.

Defining a healthy subject is not easy. Diverse criteria underlying the concept of wellness can be implied. The Royal College of Physicians has defined the healthy volunteer as an "individual who is not known to suffer any significant illness relevant to the suggested study, who should be within the normal range of body measurements. In addition, the mental state of healthy volunteers is such that he is able to understand and give valid consent to the study [4]. The EMEA guideline also proposes a general definition of healthy volunteer for studies aimed at assessing pharmacokinetics: "healthy, adult volunteers, in well-defined and controlled conditions" [5]. This definition implies that the selection of healthy volunteers is conducted by enrolling subjects without relevant pathologies and with organ functions, such as heart, liver and kidney, in the normal range.

Such general definitions of healthy volunteer, allow wide margins of discretion. A control group may include individuals similar to the trial group in all features that affect the results except for the (treatment/intervention) of interest. On the basis of comparability to the target persons or the persons at risk, controls are carefully selected. This group is critical to determine a treatment or intervention, also aiding in the assessment of efficacy and safety. A control group distinguishes results produced by the treatment or intervention of interest from those caused by other factors, for example normal course of disease [6]. In designing a clinical trial, the choice of control group is always a serious decision, because the choice affects the inferences derived from the trial [7].

\section{Objectives}

1. To calculate the prevalence rate of isolated single laboratory test abnormality.

2. To calculate the prevalence rate of joint laboratory tests abnormalities in each test domain.

3. To agree on the statistical (probability) definition of healthy control according to the result of multiple laboratory tests in the immunologic domain(s).

4. To calculate the prevalence rate of a subject being acceptable as healthy control among those appearing to be so in each laboratory test domain and in general.

\section{Material and Methods}

A total of 217 apparently healthy adult subjects were enrolled in the current study during the period of 3 months extending from March to June 2016. The sample was randomly selected from apparently healthy individuals accompanying patients attending the outpatient clinic in a tertiary referral hospital. A systematic random sample of 4 subjects was selected daily from the list of clinic attendants during the 5 working days of each week. The targeted patient was approached and his accompanying adult was asked for his consent to participate in the study. If the targeted subject had no accompanying adult, that subject did not fulfill the inclusion criteria, or the consent was denied, then the next in the list was approached. The age of participants ranged between 18 and 69 years with a mean \pm SD of $32 \pm$ 13 years.

Information related to the health status were recorded. Anthropometric measurements (height, weight) were assessed and blood presser was measured using a standard mercury sphygmomanometer. Body temperature was measured using electronic thermometer. Medical history of hypertension, diabetes, chronic diseases and risk factors such as surgical 


\section{Open Access Journal of Pharmaceutical Research}

operation and previous admission into hospital was also looked for.

A sample of venous blood $(10 \mathrm{~mm})$ was collected in the morning after an overnight fasting, and then subdivided into tow portions. The first one is used for the measurements of hematological indices by using EDTA was as an anticoagulant for preforming complete blood picture test, whereas the second part of blood samples were centrifuged in plane tubes at 3000X rpm for $15 \mathrm{~min}$. Aliquots were separated and prepared for storage $\left(-20^{\circ} \mathrm{C}\right)$ until further analysis.

Lipid profile including Total cholesterol (TC), triglycerides, and high -density lipoprotein cholesterol (HDL-C) were measured with commercially available enzymatic colorimetric kits (from biolabo, France). Serum low density lipoprotein LDL-cholesterol (LDL-C) was calculated according to the Friedewald's formula. This equation calculates the concentration of LDL-C based upon the presence of total cholesterol, HDL and triglyceride levels. In addition to biochemical related tests for serum alanine transferase, urea and creatinine.

LDL=total cholesterol-HDL- (triglycerides/5).

Immunological related tests including serum total immunoglobulin Ig (IgM, IgA, IgG) from (LTA, Italia), complement test $(\mathrm{C} 3, \mathrm{C} 4)$ from (LTA, Italia) were measured by using immune-diffusion test. Rheumatoid factor by using agglutination test (spinreact, Spain), serum levels of high sensitive $\mathrm{C}$ reactive protein (Hs-CRP) and interleukin 1 beta (IL-1 B) were measured by applying the Enzyme Linked ImmunoSorbent Assay (ELISA) technology, from (Labor Diagnostika Nord(LDN), Germany, Diaclone(France).respectivly.

\section{Statistical Analysis}

Data were translated into a computerized database and then was examined for errors using range and logical data cleaning methods, and inconsistencies were identified and corrected. Statistical analyses were done using IBMSPSS version 23 computer software (IBM Statistical Package for Social Sciences) in association with Microsoft Excel. Frequency distributions for selected variables were done first.

\section{Results}

The results presented in this research were based on the analysis of 217 apparently healthy control subjects.
Very young adults constituted $43.3 \%$ of the sample, while those 50 years and older were $14.3 \%$. Gender composition was almost equal with females comprising $52.5 \%$ of the study sample. Slightly more than half of the studied subjects $(53.5 \%)$ were of normal BMI, while only $14.3 \%$ were obese. Smokers were $28.1 \%$ of the sample. A positive history of hospital admission and surgical intervention was observed in $27.6 \%$ of subjects. In addition, a positive family history of DM, hypertension and RA was observed in $2.8,15.7$ and $16.6 \%$ of study sample respectively, table 1 .

\begin{tabular}{|c|c|c|}
\hline & $\mathbf{N}$ & $\%$ \\
\hline \multicolumn{3}{|l|}{ Age group (years) } \\
\hline very young adults $(<25)$ & 94 & 43 \\
\hline young adults (25-49) & 92 & 42 \\
\hline middle age (50-69) & 31 & 14 \\
\hline Total & 217 & 100 \\
\hline \multicolumn{3}{|l|}{ Gender } \\
\hline Female & 114 & 53 \\
\hline Male & 103 & 48 \\
\hline Total & 217 & 100 \\
\hline \multicolumn{3}{|l|}{ BMI (Kg/m2)-categories } \\
\hline Normal $(<25)$ & 116 & 54 \\
\hline Overweight (25-29.9) & 70 & 32 \\
\hline Obese $(30+)$ & 31 & 14 \\
\hline Total & 217 & 100 \\
\hline \multicolumn{3}{|l|}{ Smoking habit } \\
\hline Non smoker & 156 & 72 \\
\hline Smoker & 61 & 28 \\
\hline Total & 217 & 100 \\
\hline $\begin{array}{l}\text { Positive Past history of hospital admission } \\
\qquad(\mathrm{N}=217)\end{array}$ & 60 & 28 \\
\hline $\begin{array}{c}\text { Positive Past history of surgical } \\
\text { intervention }(\mathrm{N}=217)\end{array}$ & 60 & 28 \\
\hline Positive Family history of DM (N=217) & 6 & 2.8 \\
\hline $\begin{array}{l}\text { Positive Family History of Hypertension } \\
\qquad(\mathrm{N}=217)\end{array}$ & 34 & 16 \\
\hline $\begin{array}{l}\text { Positive Family history of Rheumatoid } \\
\text { arthritis ( }=217)\end{array}$ & 36 & 17 \\
\hline
\end{tabular}

Table 1: Description of study sam.

\section{Count of Abnormal Test Components for a Specific Test Domain}

As shown in table 2, the count of abnormal test values that belongs to a specific test domain was studied. The prevalence rate of apparently healthy individuals with a completely normal lipid profile (composed of 5 test components) was $25.3 \%$, while those with at least two abnormal test components was $34.1 \%$. 
Open Access Journal of Pharmaceutical Research

\begin{tabular}{|c|c|c|c|c|}
\hline & & $\mathbf{N}$ & $\%$ & Cumulative \% \\
\hline \multirow[t]{8}{*}{1} & Count of abnormal lipid parameters (5) & & & \\
\hline & 5 & 8 & 3.7 & 3.7 \\
\hline & 4 & 4 & 1.8 & 5.5 \\
\hline & 3 & 29 & 13 & 18.9 \\
\hline & 2 & 33 & 15 & 34.1 \\
\hline & 1 & 88 & 41 & 74.7 \\
\hline & 0 & 55 & 25 & 100 \\
\hline & \begin{tabular}{|c|} 
Total \\
\end{tabular} & 217 & 100 & \\
\hline \multirow[t]{7}{*}{2} & fount of abnormal biochemichal test parameters (4) & & & \\
\hline & 4 & 1 & 0.5 & 0.5 \\
\hline & 3 & 12 & 5.5 & 6 \\
\hline & 2 & 50 & 23 & 29.1 \\
\hline & 1 & 86 & 40 & 68.7 \\
\hline & 0 & 68 & 31 & 100 \\
\hline & $\begin{aligned} \text { Total } \\
\end{aligned}$ & 217 & 100 & \\
\hline \multirow[t]{7}{*}{3} & Count of abnormal WBC cell count parameters (6) & & & \\
\hline & 4 & 1 & 0.5 & 0.5 \\
\hline & 3 & 7 & 3.2 & 3.7 \\
\hline & 2 & 27 & 12 & 16.1 \\
\hline & 1 & 58 & 27 & 42.8 \\
\hline & 0 & 124 & 57 & 100 \\
\hline & Total & 217 & 100 & 200 \\
\hline \multirow[t]{10}{*}{4} & Count of abnormal RBC related parameters (7) & & & \\
\hline & 7 & 1 & 0.5 & 0.5 \\
\hline & 6 & 9 & 4.1 & 4.6 \\
\hline & 5 & 11 & 5.1 & 9.7 \\
\hline & 4 & 17 & 7.8 & 17.5 \\
\hline & 3 & 35 & 16 & 33.6 \\
\hline & 2 & 70 & 32 & 65.9 \\
\hline & 1 & 49 & 23 & 88.5 \\
\hline & 0 & 25 & 12 & 100 \\
\hline & Total & 217 & 100 & \\
\hline \multirow[t]{5}{*}{5} & Count of abnormal platelets parameters (2) & & & \\
\hline & 2 & 7 & 3.3 & 3.3 \\
\hline & 1 & 142 & 65 & 68.7 \\
\hline & 0 & 68 & 31 & 100 \\
\hline & Total & 217 & 100 & \\
\hline \multirow[t]{10}{*}{6} & Count of abnormal Hematologic parameters (16) & & & \\
\hline & 13 & 1 & 0.5 & 0.5 \\
\hline & 10 & 2 & 0.9 & 1.4 \\
\hline & 9 & 2 & 0.9 & 2.3 \\
\hline & 8 & 2 & 0.9 & 3.2 \\
\hline & 7 & 17 & 7.8 & 11 \\
\hline & 6 & 12 & 5.5 & 16.5 \\
\hline & 5 & 31 & 14 & 30.8 \\
\hline & 4 & 50 & 23 & 53.9 \\
\hline & 3 & 52 & 24 & 77.9 \\
\hline
\end{tabular}




\section{Open Access Journal of Pharmaceutical Research}

\begin{tabular}{|c|c|c|c|c|}
\hline & 2 & 27 & 12 & 90.3 \\
\hline & 1 & 19 & 8.8 & 99.1 \\
\hline & 0 & 2 & 0.9 & 100 \\
\hline & Total & 217 & 100 & \\
\hline \multirow[t]{9}{*}{7} & Count of abnormal immunological parameters (9) & & & \\
\hline & 6 & 2 & 1 & 1 \\
\hline & 5 & 3 & 1.4 & 2.4 \\
\hline & 4 & 12 & 5.8 & 8.2 \\
\hline & 3 & 29 & 14 & 22.2 \\
\hline & 2 & 49 & 24 & 45.9 \\
\hline & 1 & 93 & 45 & 90.8 \\
\hline & 0 & 19 & 9.2 & 100 \\
\hline & Total & 207 & 100 & \\
\hline \multirow[t]{25}{*}{8} & Count of all abnormal tests & & & \\
\hline & 29 & 1 & 0.5 & 0.5 \\
\hline & 23 & 2 & 1 & 1.5 \\
\hline & 22 & 1 & 0.5 & 2 \\
\hline & 21 & 3 & 1.4 & 3.4 \\
\hline & 20 & 2 & 1 & 4.4 \\
\hline & 19 & 2 & 1 & 5.4 \\
\hline & 18 & 8 & 3.9 & 9.3 \\
\hline & 17 & 8 & 3.9 & 13.2 \\
\hline & 16 & 7 & 3.4 & 16.6 \\
\hline & 15 & 16 & 7.7 & 24.3 \\
\hline & 14 & 15 & 7.2 & 31.5 \\
\hline & 13 & 18 & 8.8 & 40.3 \\
\hline & 12 & 10 & 4.8 & 45.1 \\
\hline & 11 & 27 & 13 & 58.1 \\
\hline & 10 & 18 & 8.7 & 66.8 \\
\hline & 9 & 21 & 10 & 76.9 \\
\hline & 8 & 9 & 4.3 & 81.2 \\
\hline & 7 & 15 & 7.2 & 88.4 \\
\hline & 6 & 4 & 1.9 & 90.3 \\
\hline & 5 & 13 & 6.3 & 96.6 \\
\hline & 4 & 1 & 0.5 & 97.1 \\
\hline & 3 & 4 & 1.9 & 99 \\
\hline & 2 & 2 & 1 & 100 \\
\hline & Total & 207 & 100 & \\
\hline
\end{tabular}

Table 2: The relative frequency of abnormal test components in each test domain.

The prevalence rate of apparently healthy individuals with a completely normal biochemistry domain tests (composed of 4 test components) was $31.3 \%$, while those with at least two abnormal test components in this domain was $29.1 \%$, table 2 .

The prevalence rate of apparently healthy individuals with a completely normal WBC cell count domain tests (composed of 4 test components) was 57.2\%, while those with at least two abnormal test components in this domain had a prevalence rate of $16.1 \%$. The prevalence rate of a completely normal RBC related domain tests (composed of 7 test components) was $11.5 \%$, while those with at least two abnormal test components in this domain was $65.9 \%$, table 3 . 


\section{Open Access Journal of Pharmaceutical Research}

\begin{tabular}{|c|c|c|c|}
\hline $\begin{array}{c}\text { Count of } \\
\text { component tests }\end{array}$ & Test Domain & $\begin{array}{c}\text { Probability of having at least one } \\
\text { abnormal component test in a } \\
\text { perfectly healthy person }\end{array}$ & $\begin{array}{c}\text { Probability of having at least two } \\
\text { abnormal component tests in a } \\
\text { perfectly healthy person }\end{array}$ \\
\hline 5 & Blood Lipids profile & $25 \%$ & $1 \%$ \\
\hline 4 & Biochemistry tests & $20 \%$ & $1.20 \%$ \\
\hline 6 & Leucocyte counts & $20 \%$ & $1.50 \%$ \\
\hline 7 & RBC count and indices & $35 \%$ & $1.70 \%$ \\
\hline 2 & Platelets & $10 \%$ & $0.50 \%$ \\
\hline 16 & Overall Hematologic & $80 \%$ & $4 \%$ \\
\hline 8 & domain & $40 \%$ & $2 \%$ \\
\hline
\end{tabular}

Table 3: The probability of having at least one abnormal component and two abnormal components in each of seven test domains in an assumed healthy person.

The prevalence rate of apparently healthy individuals with a completely normal platelets domain tests (composed of 2 test components) was $31.3 \%$. The prevalence rate of a completely normal hematologic

domain tests (composed of 16 test components) was $8.8 \%$, while $90.3 \%$ of the study sample had at least two abnormal test components in this domain, table 4.

\begin{tabular}{|c|c|c|c|}
\hline $\begin{array}{c}\text { Considering two or more domain } \\
\text { tests }\end{array}$ & $\begin{array}{c}\text { First Domain } \\
\text { probability of } \\
\text { having a single } \\
\text { abnormal test }\end{array}$ & $\begin{array}{c}\text { Second Domain } \\
\text { probability of having a } \\
\text { single abnormal test }\end{array}$ & $\begin{array}{c}\text { Probability of having at least one } \\
\text { abnormal component test in a } \\
\text { perfectly healthy person }\end{array}$ \\
\hline $\begin{array}{c}\text { (Overall Hematologic domain X } \\
\text { Immunologic tests) X Blood lipids } \\
\text { profile }\end{array}$ & $32.00 \%$ & $25.00 \%$ & $8.00 \%$ \\
\hline $\begin{array}{c}\text { Ovverall Hematologic domain X } \\
\text { Immunologic tests) X Biochemistry } \\
\text { tests }\end{array}$ & $32.00 \%$ & $20.00 \%$ & $6.40 \%$ \\
\hline $\begin{array}{c}\text { Overall Hematologic domain X } \\
\text { Biochemistry tests }\end{array}$ & $80.00 \%$ & $20.00 \%$ & $16.00 \%$ \\
\hline $\begin{array}{c}\text { Overall Hematologic domain X } \\
\text { Immunologic tests }\end{array}$ & $80.00 \%$ & $40.00 \%$ & $32.00 \%$ \\
\hline $\begin{array}{c}\text { Overall Hematologic domain X RBC } \\
\text { count and indices }\end{array}$ & $40.00 \%$ & $35.00 \%$ & $14.00 \%$ \\
\hline $\begin{array}{c}\text { Immunologic tests X Biochemistry } \\
\text { tests }\end{array}$ & $40.00 \%$ & $20.00 \%$ & $8.00 \%$ \\
\hline $\begin{array}{c}\text { Immunologic tests X Blood Lipid } \\
\text { profile tests }\end{array}$ & $40.00 \%$ & $25.00 \%$ & $9.00 \%$ \\
\hline $\begin{array}{c}\text { Leucocyte counts X RBC count and } \\
\text { indices }\end{array}$ & $20.00 \%$ & $35.00 \%$ & $7.00 \%$ \\
\hline
\end{tabular}

Table 4: The probability of having at least one abnormal component in each domain of a combination of two or three test domains of an assumed healthy person.

The prevalence rate of apparently healthy individuals with a completely normal immunologic domain tests (composed of 9 test components) was $9.2 \%$ only, while those with at least two abnormal test components in this domain had a prevalence rate of $45.9 \%$.
None of the tested individuals in the current study sample had a completely normal profile in all the performed tests. The healthiest individual in the current sample had at least two abnormal test values. More than three quarters of the current sample (76.9\%) had at least nine abnormal tests, table 2 . 


\section{Probability of Being Healthy In a Specific Test Domains in an Apparently Healthy Individual}

Based on the statistical hypothesis that an apparently healthy individual may have a single abnormal test component in a test domain and still qualifies as healthy control, we can calculate how common is the finding of a healthy control individual in apparently health people, table 5. Using the platelets test domain one expects $96.8 \%$ of study sample to qualify as a healthy control, while referring to RBC related tests only $34.1 \%$ succeed to qualify as really healthy. The final decision about an apparently healthy individual being acceptable as healthy control based on all the test domains used in the current study is only applicable to only $13.4 \%$ of the current study sample. This probability is expected to range between $8.9 \%$ and $17.9 \%$ in the reference population $(95 \%$ confidence interval).

\begin{tabular}{|c|c|c|c|}
\hline & $\mathbf{N}$ & $\%$ & 95\% confidence interval \\
\hline \multicolumn{4}{|l|}{$\begin{array}{l}\text { Accepted as normal (none or only one randomly abnormal } \\
\text { component) } \mathrm{N}=217\end{array}$} \\
\hline platelets parameters & 210 & 97 & (94.5 to 99.1$)$ \\
\hline Leucocyte count & 182 & 84 & (79 to 88.8$)$ \\
\hline Overall Hematologic parameters (platelets, Leucocyte and RBC domains) & 170 & 78 & (72.8 to 83.8$)$ \\
\hline biochemical test & 154 & 71 & (64.9 to 77.1$)$ \\
\hline lipid profile & 143 & 66 & (59.6 to 72.2$)$ \\
\hline immunological parameters $(\mathrm{N}=207)$ & 112 & 54 & (47.5 to 60.7$)$ \\
\hline RBC related parameters & 74 & 34 & (27.8 to 40.4$)$ \\
\hline Accepted as normal control based on multiple testing ( $\mathrm{N}=217)$ & 29 & 13 & (8.9 to 17.9 ) \\
\hline
\end{tabular}

Table 5: The probability of having none or only one randomly abnormal component for each test domain in a healthy individual.

\section{Discussion}

"An event, condition, or characteristic is not a cause by itself as an intrinsic property it possesses in isolation, but as part of a causal contrast with an alternative event, condition, or characteristic" [8-10]. The Neyman-Rubin causal model also known as the "potential outcome or counterfactual model" established the general framework for using the control or reference group in both observational and experimental types of analytic studies $[11,12]$. Referring to this definition of a cause or risk factor one can understand the critical role for selecting a control group in any analytic study. The choice of a control group in any analytic study can be the single most important factor in deciding the outcome of the study. It can change the possible conclusion of a study from no association between an exposure (explanatory variable, risk factor or a possible cause) and an outcome (response variable or disease status) to a strong effect or association.

Researchers in the field of medical or biology sciences often need to enroll a group healthy control subjects in their studies. The term "apparently healthy control" is more suitable than "healthy control", since any subject who is not currently complaining, has no annoying symptoms or obvious clinical features can be included in such a comparison group. Such a selected group is expected to depart from the reference values of many tests or biologic measurements. This departure from normality of a control group tends to conceal and confound any real differences in the cases or intervention group.

The present study used a statistical framework to screen healthy subjects from a group of "apparently healthy" individuals. Some tests provide a simple yes or no (positive or negative, reactive or non-reactive) interpretation. Other tests are quantitative. A typical lab report will provide such test results followed by a reference range [13]. The term "reference range" is preferred over "normal range" because the reference population can be clearly defined. Rather than implying that the test results are being compared with some vague definition of "normal," the reference range means the results are being considered in the most relevant context. By definition, 1 out of 20 (or 5\%) results will fall outside the established reference range with specimens taken from a random sample of healthy individuals $[14,15]$.

The current study evaluated the probability of being qualified as "Healthy Control" with selected laboratory tests within internationally agreed reference range. A total of 34 different tests were done per study participant. 


\section{Open Access Journal of Pharmaceutical Research}

These tests belonged to lipid, biochemistry, hematology and immunology domains. If all the domains are needed to define a healthy control only $13.4 \%$ of the apparently healthy controls would qualify for a laboratory verified healthy controls. However most of the analytic studies would require a laboratory verified healthy control in only one domain of tests. More than three quarters of randomly selected apparently healthy controls would qualify for inclusion as hematologically verified healthy controls. This probability decline to slightly more than a half for immunologically verified healthy controls. Finally, a lipid profile or biochemistry verified healthy controls would be obtained with a probability of around two thirds among a random sample of apparently healthy controls. In conclusion it can be said that a very considerable proportion of population who appear to be healthy , are not in reality, accordingly not all apparently healthy controls are qualified as eligible control.

\section{References}

1. Grasbeck R (1983) Reference value philosophy. Bulletin of Experimental Biology and Medicine 8(1-2): 1-11.

2. Henny J, Petersen PH (2004) Reference values: from philosophy to a tool for laboratory medicine. Clin Chem Lab Med. 42(7): 686-691.

3. Horowitz G, Altaie S, Boyd J, Ceriotti F, Garg U, et al. (2008) Defining, Establishing, and Verifying Reference Intervals in the Clinical Laboratory; Approved Guideline. Clinical and Laboratory Standards Institute 28(30): 1-12.

4. (1986) Research on healthy volunteers. A report of the Royal College of Physicians. J R Coll Physicians Land 20(4): 243-257.

5. Pasqualetti G, Gori G, Blandizzi C, Del Tacca M (2010) Healthy volunteers and early phases of clinical experimentation. Eur J Clin Pharmacol 66(7): 647653.
6. Malay S, Chung KC (2012) The choice of controls for providing validity and evidence in clinical research. Plast Reconstr Surg 130(4): 959-965.

7. EMA (1998) Committee for Proprietary Medicinal Products (CPMP). European Medicines Agency's pp: $1-11$

8. Rubin DB (1974) Estimating causal effects of treatments in randomized and nonrandomized studies. Journal of educational Psychology 66(5): 688-701.

9. Maldonado G, Greenland S (2002) Estimating causal effects. International journal of epidemiology 31(2): 422-429.

10. Iacus SM, King G, Porro G (2019) A theory of statistical inference for matching methods in causal research. Political Analysis 27(1): 46-68.

11. Rubin DB (2005) Causal inference using potential outcomes: Design, modeling, decisions. Journal of the American Statistical Association 100(469): 322-331.

12. Valente MJ, Rijnhart JJ, MacKinnon DP (2019) Causal direct and indirect effects: The link between traditional logistic regression and the potential outcomes framework for binary outcomes. SPR.

13. Jørgensen LG, Brandslund I, Petersen PH (2004) Should we maintain the 95 percent reference intervals in the era of wellness testing? A concept paper. Clin Chem Lab Med 42(7): 747-751.

14. Phillips P (2009) Pitfalls in interpreting laboratory results. Aust Prescr 32: 43-46.

15. Monday J (2018) New Diagnostic Advancements: Syndromic Approach to Diagnostic Testing. 